\title{
Healing difficult wounds: A primer for advanced practice registered nurses
}

\author{
Karen C. Lyon \\ Nelda C. Stark College of Nursing, Texas Woman's University, Houston Center, United States \\ Correspondence: Karen C. Lyon. Address: 6700 Fannin St., Houston, TX 77030, United States. \\ Email: KLyon2@mail.twu.edu
}

Received: April 2, 2013

Accepted: August 1, 2013

Online Published: August 11, 2013

DOI : $10.5430 /$ cns.v1n3p77

URL: http://dx.doi.org/10.5430/cns.v1n3p77

\begin{abstract}
There are a variety of factors that cause wounds to become chronic and non-healing, all of which can be categorized as either local or systemic. Local factors include ischemia, infection, edema and pressure while systemic factors include co-morbidities such as diabetes, cardiovascular and peripheral vascular failure, neurological compromise, immobility issues, and renal insufficiency/failure. This paper describes those factors from the standpoint of an actual clinical situation with a particular emphasis on the elderly and influences that make healing wounds difficult in this population.

The tools available to heal chronic wounds are also described with emphasis on skills for the advanced practice registered nurse (APRN), primarily the nurse practitioner and clinical nurse specialist. From comprehensive wound centers to sharp debridement, hyperbaric oxygen therapy, and vacuum assisted closure, advanced wound therapies are described. Finally, competencies expected of APRNs working with this population including direct care, consultation and education of patients, family members and other wound care staff are also discussed.
\end{abstract}

\section{Key Words}

Non-healing wound, Debridement, Vacuum-assisted closure device, Hyperbaric oxygen therapy (HBO), Wound care competencies

\section{I ntroduction}

From the moment we are born, our skin is exposed to physical and environmental assaults that can have temporary or permanent consequences. In the elderly population, the effects of these attacks, coupled with chronic co-morbid conditions can transform an acute skin break into a chronic, non-healing wound. Using a clinical case design, this discussion describes wound care issues for the elderly and the advanced therapies we have for managing them.

\section{Case presentation}

Mrs. X is an 86-year-old woman admitted to a hospital-based, outpatient wound center following a right below-the-knee amputation (BKA). The amputation resulted after previous hospitalization and routine wound care were unsuccessful in healing chronic, lower extremity wounds. Her medical history includes coronary artery disease with placement of stents, hypertension, and hypercholesterolemia. Previous surgeries include coronary artery bypass grafts (CABG) in 2007 and 
2010 and bilateral lower extremity revascularization. Mrs. X has never smoked and does not have diabetes. Other than those two anomalies, however, she presents with the typical morbidities of patients with chronic, non-healing wounds including peripheral vascular insufficiency, extremes of age and nutritional deficiencies.

Using Mrs. X's history, the case study attempts to identify the variety of factors that cause wounds to become chronic and non-healing with a particular focus on the elderly population. Problem wounds cannot be treated successfully unless they are recognized early, diagnosed appropriately and managed aggressively. With over 800,000 active wound cases costing over $\$ 5$ billion annually in the United States, both prevention and appropriate treatment are financial imperatives ${ }^{[3,4,12]}$.

\section{Pathogenesis of wound healing failure}

In the healthy patient without co-morbid conditions, healing proceeds through the normal processes of wounding, hemostasis, inflammation, angiogenesis, granulation tissue formation, epithelialization, and remodeling ${ }^{[5,7,9]}$. Problem wounds do not proceed through that orderly process. Why do some wounds become chronic and non-healing? There are a variety of factors including ischemia, infection, edema, scarring, trauma, pressure, foreign bodies, and local malignancy. Complicating these local factors is the presence of systemic co-morbidities such as diabetes mellitus, connective tissue disease, renal failure, hereditary and immunological disorders, and hepatic failure. These factors are exacerbated by the aging process. As we age, the functions of the skin as a vital organ decline. The effects of repetitive, physical trauma, chronic disease, malnutrition and diminished blood and oxygen supply are synergistic and the cost in human suffering is incalculable. Additionally, the edema of chronic venous insufficiency (CVI) often seen in the elderly aggravates the already diminished arterial flow and oxygen delivery to chronic wounds. The current hypotheses for how venous insufficiency complicates lower leg wounds include theories concerning the deposition of fibrin in response to the aforementioned venous hypertension, damage to endothelial cells with release of inflammatory mediators and trapping of growth factors and matrix components ${ }^{[10]}$. Together, the factors of peripheral arterial disease and edema coupled with trauma and infection interfere with the delivery of oxygen to the skin and subsequent tissue repair and regeneration. In Mrs. X's case, these factors were a prescription for disaster and served as critical events leading to below-the-knee amputation and subsequent wound healing failure of the incision site.

Impaired nutrition is another significant factor in the development or deterioration of wounds in elderly patients. These patients are often protein-calorie malnourished and deficient in adequate minerals and vitamins. Advanced practice nurses should have a high index of suspicion for malnutrition in any patient with chronic illnesses, lack of familial or social support mechanisms, major trauma or gastrointestinal or neurological disorders that impair oral intake. Vitamin and mineral deficiencies should also be assessed including Vitamins A, C, and E and zinc, all of which play an important role in normal wound healing ${ }^{[1]}$. In Mrs. X's case, the Adult CNS assessed age (86), social isolation, polypharmacy, and chronic medical co-morbidities to be factors for malnutrition in the patient. She consulted with the dietitian at the skilled nursing facility to establish an appropriate dietary plan of care including monthly assessments of weight change, maintenance of a dietary journal by the nursing staff, increased intake and variety of vegetables, fruits and fluids, assistance with feeding, and protein supplement shakes mid-morning and mid-afternoon.

\section{The wound care tool box}

\subsection{The wound care center}

Mrs. X had the benefit of being seen in a wound care center where advanced therapies are available and care is coordinated among specialists including surgeons, internists, infectious disease specialists, nurse practitioners and registered nurses with specialized training in wound care. The availability of advanced treatment modalities and clinical expertise in a dedicated wound center can positively influence wound healing because experts are available to confirm clinical 
judgment. The treatment plan may call for surgical intervention, infection control, nutritional counseling, orthotics/prosthetics, compression therapy, hyperbaric treatments, diabetic counseling and family intervention, all of which must be provided by specialists who understand the dynamic process of wound healing. In the absence of a formal wound center, the APRN must manage chronic wounds according to the mechanisms underlying their development. This would begin with differential diagnosis; the typical venous ulcer presents as a painful lesion on the lower leg with periwound hyperpigmentation and lipodermatosclerosis ${ }^{[11]}$. This should be accompanied by palpation of the pedal ulcers to assess for co-morbid arterial disease since nearly $1 / 4$ of venous ulcers have an arterial component ${ }^{[11]}$. However, this assessment is often difficult since the edema associated with venous insufficiency often makes this assessment challenging. Finally, if the ulcer has been present for longer than 6 months duration and has not responded to therapy, the differential diagnosis should also include biopsy since squamous or basal cell carcinomas have been frequently reported in otherwise "classic" venous ulcers ${ }^{[11]}$. Treatment will include controlling edema and venous hypertension with compression therapy. In diabetics, the best management is prevention of wounds including inspection for callus formation, assessment for neuropathy, annual foot screening and patient education regarding proper selection of footwear, daily foot inspections and blood glucose control. Management of pressure ulcers revolves around prevention and treatment. Prevention and treatment protocols can be found in the National Guideline Clearinghouse ${ }^{[12]}$.

\subsection{Debridement}

Surgical interventions include aggressive debridement to convert chronic, non-healing wounds into acute, inflammatory wounds. Debridement allows for the removal of devitalized tissue and foreign material. Additionally, it removes senescent cells that impede healing and interfere with the deposition of growth factors. While it can be accomplished through the use of autolytic debriding agents and mechanical instrumentation, the quickest and most effective method is sharp debridement with a scalpel whenever significant necrotic or gangrenous tissue is present in the wound ${ }^{[8,10]}$. The CNS saw Mrs. X every week and provided sharp debridement as necessary. The clinical photographs below demonstrate Mrs. X's wound along the BKA site when she was admitted in March 2011 and then post-sharp debridement that same month. After removal of the sutures, there was some wound dehiscence on the lateral aspect of the amputation site. This area has been sharply debrided by the APRN using a \#15 blade (see Figure 1). The periwound area demonstrates significant decrease in erythema and inflammation post debridement and initiation of hyperbaric oxygen treatments (HBO).

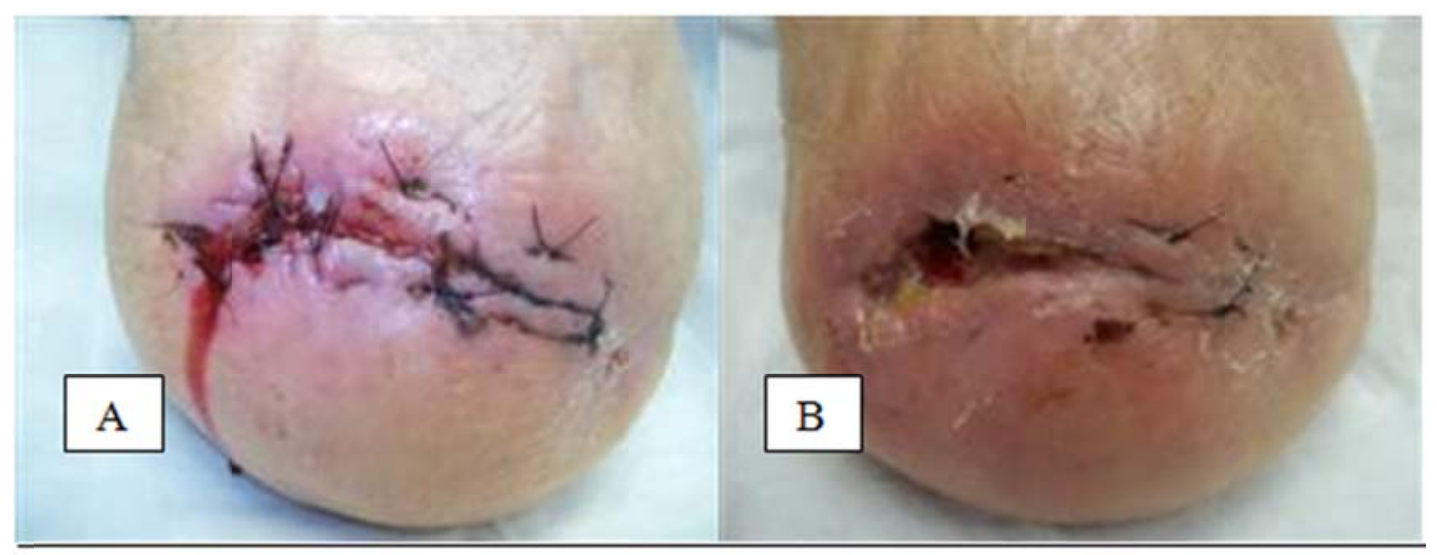

Figure 1. Pre and post debridement photographs

A. Initial post-BKA suture site. B. Post removal of sutures and sharp debridement

\subsection{Hyperbaric Oxygen Therapy (HBO)}

HBO therapy is defined as high dose oxygen inhalation therapy in which a patient breathes $100 \%$ oxygen $\left(\mathrm{O}_{2}\right)$ inside a pressurized hyperbaric chamber (see Figure 2) ${ }^{[9]}$. In clinical medicine, the most important effect of hyperbaric oxygen is 
to increase the partial pressure of oxygen in all the tissues of the body. At pressure higher than normal atmosphere, oxygen behaves like a drug with specific results. It is used to improve the $\mathrm{O}_{2}$ environment necessary for wound healing, diminish tissue swelling and improve circulation. Mrs. X was started on 5 day/week HBO treatments in March 2011 and concluded 36 treatments in May 2011. Her therapy utilized a monoplace chamber similar to the one pictured below. These chambers are pressurized with $100 \%$ oxygen. The standard therapy protocol includes pressurization at 2.0 ATA, equivalent to 30 pounds of atmospheric pressure/square inch for 90 minutes including 10 minute descent and 10 minute ascent ${ }^{[2]}$. At this pressure, in the presence of $100 \%$ oxygen, dissolution of oxygen in the blood plasma occurs, increasing oxygen concentration in all body tissues. Under CMS guidelines, the patient undergoing HBO therapy must be monitored at all times by a certified HBO tech and either an HBO-certified MD, APRN or PA (physician assistant). Mrs. X was monitored during her dives by the CNS or wound care physician, including $\mathrm{SpO}_{2}$ measurements through pulse oximetry, pre and post-dive blood glucose levels, and pre and post-dive ear inspections for barotrauma. Mrs. $\mathrm{X}$ 's $\mathrm{SpO}_{2}$ reading was consistently at 99\%, her blood glucose ranged from 125 - 140 and there was no indication of barotrauma on otoscopic examination.

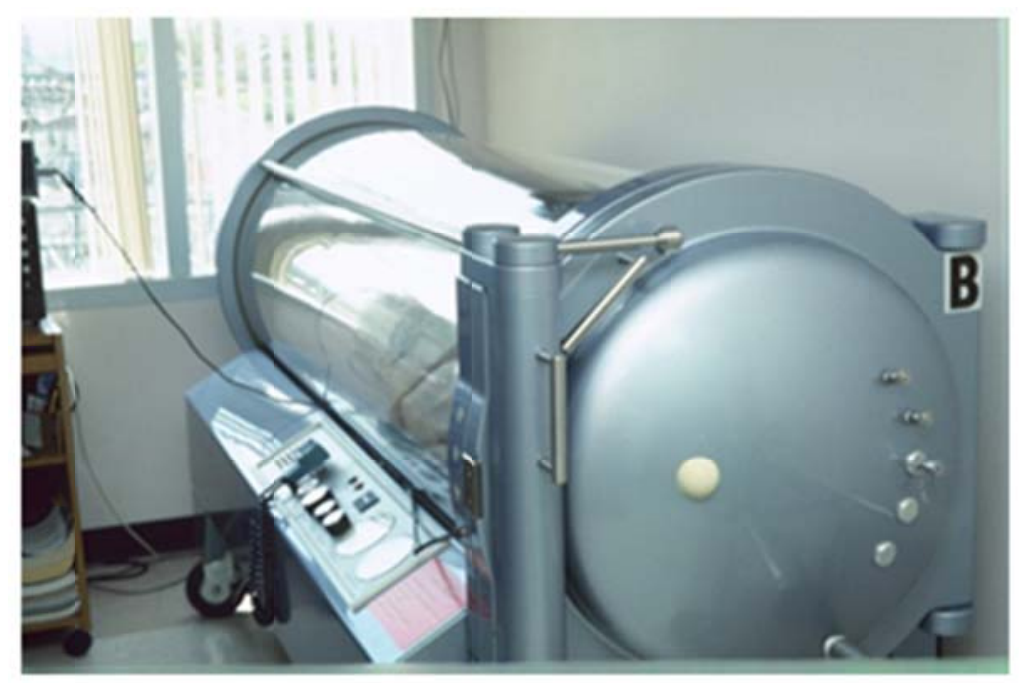

Figure 2. Monoplace hyperbaric oxygen chamber

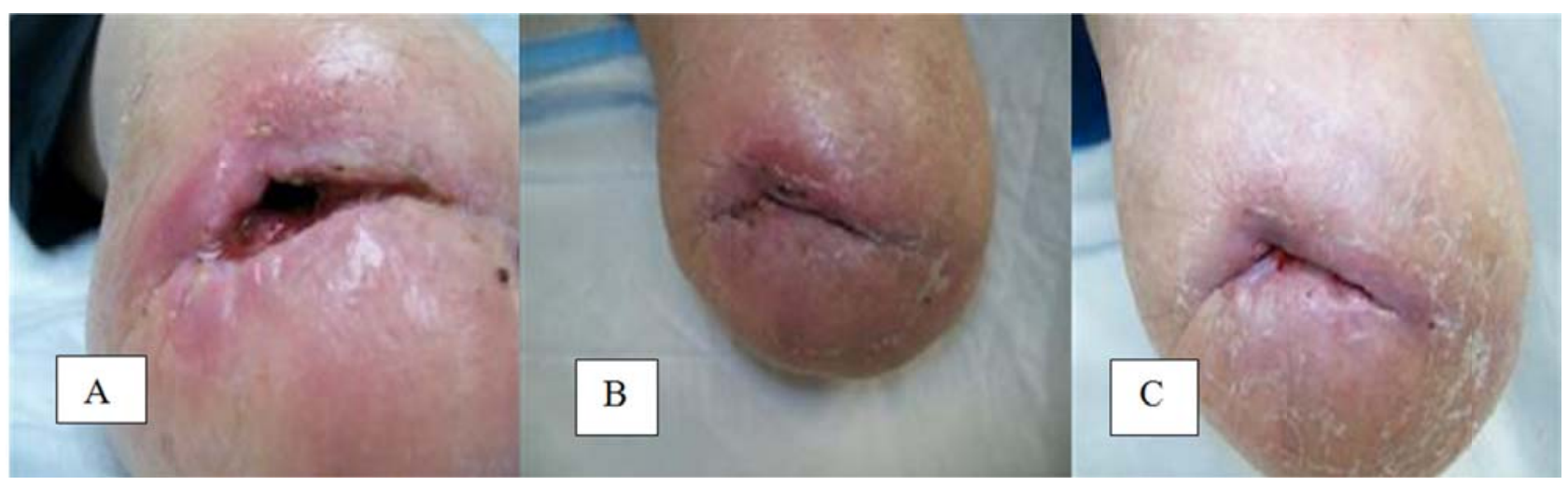

Figure 3. Progressive healing using negative pressure wound therapy

A. Wound on June 10, 2011 with NPWT. B. Wound on June 20, 2011 with NPWT. C. Wound on June 30, 2011; NPWT

\subsection{Negative Pressure Wound Therapy (NPWT)}

Wound closure assisted by negative pressure therapy is a non-invasive localized treatment modality used to promote healing. Specific uses include dehisced wounds, diabetic lower extremity wounds, pressure ulcers, flaps, and graft 
procedures. This procedure promotes healing by removing interstitial fluid, which contributes to an increase in local blood supply. Additionally, the devices assist in limiting bacterial colonization, increase the production of healing granulation tissue, enhance epithelial cell migration, and stimulate cell division within the wound margin ${ }^{[5]}$. Mrs. X was started on NPWT therapy in April 2011; her wound was surgically revised in May 2011 and she was restarted on NPWT in June 2011(see Figure 3). By the end of June, she was sufficiently healed to be fitted for a prosthesis and she was discharged healed in July.

Table 1. Differential diagnosis of chronic wounds

\begin{tabular}{|c|c|c|}
\hline $\begin{array}{l}\text { Evaluation for differential } \\
\text { diagnosis }\end{array}$ & Description & Normals \\
\hline Ankle-brachial index (ABI) & $\begin{array}{l}\text { Noninvasive test to determine blood pressure } \\
\text { differences between upper and lower extremities }\end{array}$ & $\begin{array}{l}\mathrm{ABI}=1.0 \\
\mathrm{ABI}: 0.4-0.8=\text { moderate arterial } \\
\text { insufficiency } \\
\mathrm{ABI}<0.4=\text { severe PAD }\end{array}$ \\
\hline Duplex venous scan & $\begin{array}{l}\text { Noninvasive ultrasound that detects and quantifies } \\
\text { venous reflux }\end{array}$ & Negative for stenosis or occlusion \\
\hline Biopsy & Useful for evaluating an atypical ulcer & $\begin{array}{l}\text { Negative for basal cell carcinoma, } \\
\text { melanoma, vasculitis, etc. }\end{array}$ \\
\hline Risk factors for ulceration & $\begin{array}{l}\text { Previous history of ulcers; foot deformities; loss of } \\
\text { protective sensation; limited joint mobility; obesity; } \\
\text { poor/absent glycemic control; impaired vision }\end{array}$ & \\
\hline Presence of infection & $\begin{array}{l}\text { Wound with swelling, redness, pain, malodorous } \\
\text { discharge; elevated WBC, ESR and C-reactive } \\
\text { protein; elevated blood glucose; MRI positive for } \\
\text { osteomyelitis }\end{array}$ & $\begin{array}{l}\text { WBC }=5-10,000 / \mathrm{mm}^{3} \\
\mathrm{ESR}<15-20 \mathrm{~mm} / \mathrm{hr} \\
\mathrm{CRP}=0.1 \mathrm{mg} / \mathrm{dl} \\
\text { Glucose = up to } 100 \mathrm{mg} / \mathrm{dl}\end{array}$ \\
\hline $\begin{array}{l}\text { Semmes-Weinstein } \\
\text { monofilament test }\end{array}$ & $\begin{array}{l}5.07 \text { monofilament exerts } 10 \text { gms. pressure against } \\
\text { skin when bowed into C- shape for one second }\end{array}$ & $\begin{array}{l}\text { Patients should be able to detect pressure } \\
\text { at various points along the plantar } \\
\text { surface of the foot. It they can't, they } \\
\text { have lost protective sensation. }\end{array}$ \\
\hline PVD screening & $\begin{array}{l}\text { Palpate all lower extremity pulses } \\
\text { Presence/absence of claudication } \\
\text { Lower limb angiography }\end{array}$ & \\
\hline
\end{tabular}

\section{Advanced practice competencies}

The nurse practitioner (NP) or clinical nurse specialist (CNS) for wound care is an essential member of the multidisciplinary team for wound management. Diagnosis is based on direct assessment of the wound(s) and clear differential diagnosis based on previously discussed factors as well as testing (see Table 1$)^{[5,11]}$. The ankle-brachial (ABI) index is a simple, non-invasive test for determining blood pressure differences between upper and lower extremities. An $\mathrm{ABI}<0.9$ signals arterial insufficiency and contraindicates the use of compression therapy for venous ulcers until such time as more sophisticated tests for arterial function can be performed ${ }^{[7]}$. Duplex venous scans are non-invasive ultrasound procedures that detect and quantify venous reflex. Biopsies are useful for evaluating atypical wounds such as basal cell carcinomas or malignant melanomas ${ }^{[7]}$. In addition to testing, direct care includes developing prevention strategies, assessing patient risk, minimizing environmental risk and interventions to include enzymatic debridement, mechanical 
debridement and limited sharp debridement. APRNs will also prescribe pharmacologic agents based on results of tissue cultures and bacterial sensitivities. This most commonly includes third generation cephalosporins such as ceftazidime for pseudomonas, methicillin and oxacillin for staph infections, and penicillin, erythromycin or clindamycin for group A and

B strep ${ }^{[3,11]}$. Pain control can be accomplished with medications such as oxycodone/acetaminophen (2.5/325 - 10/325) every 4-6 hours or tramadol, 50-100 mg daily for chronic pain or every 4-6 hours for rapid onset of analgesia. Selection of protective dressings, such as alginates, antimicrobials, collagens, hydrocolloids or hydrogels, will depend on the cause of the wound and the amount and type of exudate ${ }^{[7]}$. Other advanced procedures will include supervision of HBO therapy and application of skin substitutes such as Apligraf ${ }^{\circledR}$ and Dermagraft ${ }^{[7]}$.

The consultative role is also an essential skill in the advanced practice tool box. The expertise provided by a NP or CNS providing wound care in their practice contributes the best evidence and science available to assist registered nurses and other care providers with assessment, risk factor evaluation and dressing selections for patients with problem wounds.

Patient education and compliance is essential for wound healing. Staff education and mentoring is necessary to make sure that this education takes place. The current knowledge level of staff nurses related to wound risk assessment, wound prevention, wound evaluation, and wound treatment must be assessed. Principles of adult learning should be used to identify barriers to learning, evaluate current knowledge level in regards to wound management, identify staff learning styles and learning goals, and provide ongoing documentation of staff wound care skills.

Problem wounds are a major cause of human suffering and a financial drain on hospital and national resources ${ }^{[3,5,7,9,10]}$. In failing to manage these wounds with the same sense of importance given to a patient's cardiovascular, respiratory, and neurologic status, we fail our patients. The overarching goal of effective wound care in any setting should be assessing and treating systemic illness, evaluating nutritional status and correcting deficiencies, identifying and treating infections, and developing an evidence-based protocol for wound healing.

\section{References}

[1] Arnold M, Barbul A. Nutrition and wound healing. Plastic and Reconstructive Surgery, June supplement. 2006: 42S-58S.

[2] Fife CE. Hyperbaric oxygen therapy applications in wound care. In Sheffield, Smith and Fife (Eds.) Wound Care Practice. Flagstaff. AZ: Best Publishing Co. 2004.

[3] Frykberg RG. Diabetic foot disorders. A clinical practice guideline. The Journal of Foot and Ankle Surgery. Supplement 2000: 39(5): S1-60.

[4] Gist S, Ti-Matos I, Falzgraf S, Cameron S, Beebe M. Wound care in the geriatric client. Clinical Interventions in Aging, 2009; 4: 269-287.

[5] Hess CT. Clinical Guide: Skin \& Wound Care, 6th ed. Ambler, PA: Lippincott,Williams \& Wilkins; 2008.

[6] Lehman CA. Phenomena of concern to the clinical nurse specialist. In Foster and Prevost (Eds.) Advanced Practice Nursing of Adults in Acute Care. Philadelphia, PA: F.A. Davis Company; 2012.

[7] Lyon KC. Skin problems and wound care. In Foster and Prevost (Eds.) Advanced Practice Nursing of Adults in Acute Care. Philadelphia, PA: F.A. Davis Company; 2012.

[8] Sheffield PJ, Smith PS. Physiological and pharmacological basis of hyperbaric oxygen therapy. In Bakker and Cramer (Eds.) Hyperbaric Surgery Perioperative Care. Flagstaff, AZ: Best Publishing Co. 2002.

[9] Sheffield PJ, Smith PS, Fife CE. Wound Care Practice. Flagstaff, AZ: Best Publishing Co. 2004.

[10] Stillman RM. Wound care. Medscape Reference: Drugs, Diseases \& Procedures [Internet]. 2009 Sept. 29 [cited 2013 Apr 10] Available from http://emedicine.medscape.com/article/194018-overview.

[11] Weingarten MS. State-of-the-art treatment of chronic venous disease. Clinical Infectious Diseases. 2001; 32(6): 949-954. PMid:11247717 http://dx.doi.org/10.1086/319360

[12] Wound, Ostomy, and Continence Nurses Society. Guideline for management of wounds in patients with lower-extremity neuropathic disease. WOCN Clinical Practice Guideline. 2004; 3: 1-14. 